INPLASY

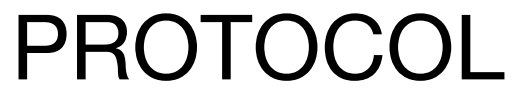

To cite: Lidong. Effect of Tai Chi on Patients with Knee Osteoarthritis: An updated Systematic Review and MetaAnalysis. Inplasy protocol 202070033. doi:

10.37766/inplasy2020.7.0033

Received: 10 July 2020

Published: 10 July 2020

Corresponding author: Wang Lidong

wldwong@outlook.com

Author Affiliation:

Nanjing University of Posts and Telecommunications

Support: None.

Review Stage at time of this submission: Data analysis.

Conflicts of interest: None.

\section{Effect of Tai Chi on Patients with Knee Osteoarthritis: An updated Systematic Review and Meta-Analysis}

Lidong, W1.

Review question / Objective: To evaluate the intervention effect of tai chi exercise on knee osteoarthritis (KOA) patients with Meta-analysis. Unlike previous reviews, we will include Chinese patients and more KOA patients.

Condition being studied: KOA patients may benefit from Tai Chi practice. As a mild, low-intensity exercise for the joints, Tai Chi USES functional exercises to enhance strength, balance, and flexibility, as well as physical therapy, so Tai Chi may be used as an adjunct to therapy for KOA patients. Currently, there have been systematic studies on the therapeutic effect of Taijiquan on KOA patients, but there are defects such as too few included studies and short intervention cycle. Therefore, this paper combines relevant reports in recent years to collect more data and carry out further research on the therapeutic effect of Taijiquan on KOA patients.

INPLASY registration number: This protocol was registered with the International Platform of Registered Systematic Review and Meta-Analysis Protocols (INPLASY) on 10 July 2020 and was last updated on 10 July 2020 (registration number INPLASY202070033).

\section{INTRODUCTION}

Review question / Objective: To evaluate the intervention effect of tai chi exercise on knee osteoarthritis (KOA) patients with Meta-analysis. Unlike previous reviews, we will include Chinese patients and more KOA patients.

Condition being studied: KOA patients may benefit from Tai Chi practice. As a mild, low-intensity exercise for the joints, Tai Chi 
USES functional exercises to enhance strength, balance, and flexibility, as well as physical therapy, so Tai Chi may be used as an adjunct to therapy for KOA patients. Currently, there have been systematic studies on the therapeutic effect of Taijiquan on KOA patients, but there are defects such as too few included studies and short intervention cycle. Therefore, this paper combines relevant reports in recent years to collect more data and carry out further research on the therapeutic effect of Taijiquan on KOA patients.

\section{METHODS}

Search strategy: ("Tai Ji"[Mesh]) OR ((Tai Chi[Title/Abstract]) OR Chi, Tai [Title/ Abstract]) OR Tai Ji Quan[Title/Abstract]) OR Ji Quan, Tai[Title/Abstract]) OR Quan, Tai Ji[Title/Abstract]) OR Taiji[Title/ Abstract]) OR Taijiquan [Title / Abstract]) OR T'ai Chi[Title/Abstract]) OR Tai Chi Chuan [Title/Abstract]) AND (randomized controlled trial[Publication Type] OR randomized[Title/Abstract] OR placebo [Title /Abstract]) AND (("Osteoarthritis, Knee"[Mesh]) OR (Knee Osteoarthritides [Title / Abstract]) OR Knee Osteoarthritis [Title/Abstract]) OR Osteoarthritides, Knee[Title/ Abstract]) OR Osteoarthritis Of Knee[Title/Abstract]) OR Knee, Osteoarthritis Of [Title/Abstract]) OR Knees, Osteoarthritis Of[Title/Abstract]) OR Osteoarthritis Of Knees[Title/Abstract])).

Participant or population: Studies of adults (18 years of age and older) with osteoarthritis of the knee were eligible. Diagnosis had to be based on valid instruments, such as the Classification Criteria of the American College of Rheumatology, 3,15 radiographic or laboratory evidence, or medical records. No further restriction regarding disease duration and intensity were applied.

Intervention: Studies that compared Tai Chi with no treatment, usual care, placebo or any active treatment were eligible. No restrictions were made regarding details of Tai Chi protocol. Co-interventions were allowed.
Comparator: Placebo or any active treatment were eligible

Study designs to be included: Only randomized controlled trials (RCTs) were considered eligible.

Eligibility criteria: Studies that compared Tai Chi with no treatment, usual care, placebo or any active treatment were eligible. No restrictions were made regarding details of Tai Chi protocol. Cointerventions were allowed.

Information sources: We will search the target study from PubMed, Embase, Scopus, Web of Science, and CNKI. There is no restriction will be imposed on the data of publication, and all studies published before April 2020 will be included. And no language restrictions on retrieval at the same time.

Main outcome(s): Pain intensity, Physical function, Stiffness, Quality of life, Safety.

Additional outcome(s): None.

Quality assessment / Risk of bias analysis: All included studies will be assessed for their methodological quality. The Cochrane Collaboration's risk of bias tool will be used (RevMan 5.3). The criteria consist of 7 items: random sequence generation, allocation concealment, blinding of participants and researchers, blinding of outcome assessment, incomplete outcome data, selective outcome reporting, and other sources of bias.

Strategy of data synthesis: Two reviewers independently extracted data on study characteristics such as participants, interventions, control conditions, cointerventions, outcome measures, and results. Disagreements were rechecked with a third reviewer and resolved by discussion. If at least five studies were available on an outcome, meta-analyses were calculated utilizing Review Manager 5 software (Version 5.3, The Nordic Cochrane Centre, Copenhagen). Standardized mean differences (SMD) with $95 \%$ confidence intervals $(\mathrm{Cl})$ were calculated as the mean 
group difference divided by the pooled standard deviation. Where no standard deviations were available, standard errors, confidence intervals, or $t$ values were used to calculate them.

Subgroup analysis: We will conduct a subgroup analysis, including scale, Intervention cycle to look for sources of heterogeneity.

Sensibility analysis: We will conduct a sensitivity analysis to see if the data of each article will affect the result.

Language: There are no language restrictions.

Country(ies) involved: All the authors are from China.

Keywords: tai chi; Osteoarthritis of the knee; Meta-analysis; Review.

Contributions of each author:

Author 1 - Wang Lidong. 